\title{
SUBAQUATIC DIGITAL ELEVATION MODELS FROM UAV-IMAGERY
}

\author{
C. Mulsow ${ }^{1}$, R. Kenner ${ }^{2}$, Y. Bühler ${ }^{2}$, A. Stoffel ${ }^{2}$, H.-G. Maas ${ }^{1}$ \\ ${ }^{1}$ Institute of Photogrammetry and Remote Sensing, Technische Universität Dresden - \\ (christian.mulsow, hans-gerd.maas)@tu-dresden.de \\ ${ }^{2}$ WSL Institute for Snow and Avalanche Research SLF, Davos Switzerland - (robert.kenner, buehler, stoffel) @slf.ch
}

\section{Commission II, WGII/9}

KEY WORDS: UAV, DEM, underwater, multi-media, bundle-adjustment

\section{ABSTRACT:}

The paper presents an approach for the generation of digital elevation models (DEMs) of underwater areas from aerial images. Standard software-products do not provide the possibility to measure correctly through refractive interfaces, such as water. Existing solutions for that problem are based on oriented images and known water levels with the DEM points determined by forward intersection based on reconstructed image ray paths (ray tracing). In this article we present an integrated procedure for image orientation as well as DEM mass point determination from aerial imagery containing both land and underwater areas. The proof of concept was done by capturing UAV imagery of shallow water areas of a high-alpine lake in the Swiss alps. In the paper the processed dataset will be presented. Furthermore, the extraction and matching of image-points observed through water are discussed. The accuracy potential as well as practical limitations of processing multimedia-data are analysed.

\section{MOTIVATION}

The talus slope at Flüelapass was the first mountain permafrost study site in Switzerland in the and the presence of ice-rich permafrost at the foot of the slope has first been by (Haeberli, 1975). Recent investigations led to new hypotheses on the geomorphological processes at the study site (Kenner et. al, 2017). One important data set for the research was a digital elevation model of the area, which also includes the bottom of a lake named Schottensee (see Figure 1). The permafrost layers are present at the slopes beside the lake but spread into the water. To survey such regions, we have to account for the two different media, air and water.

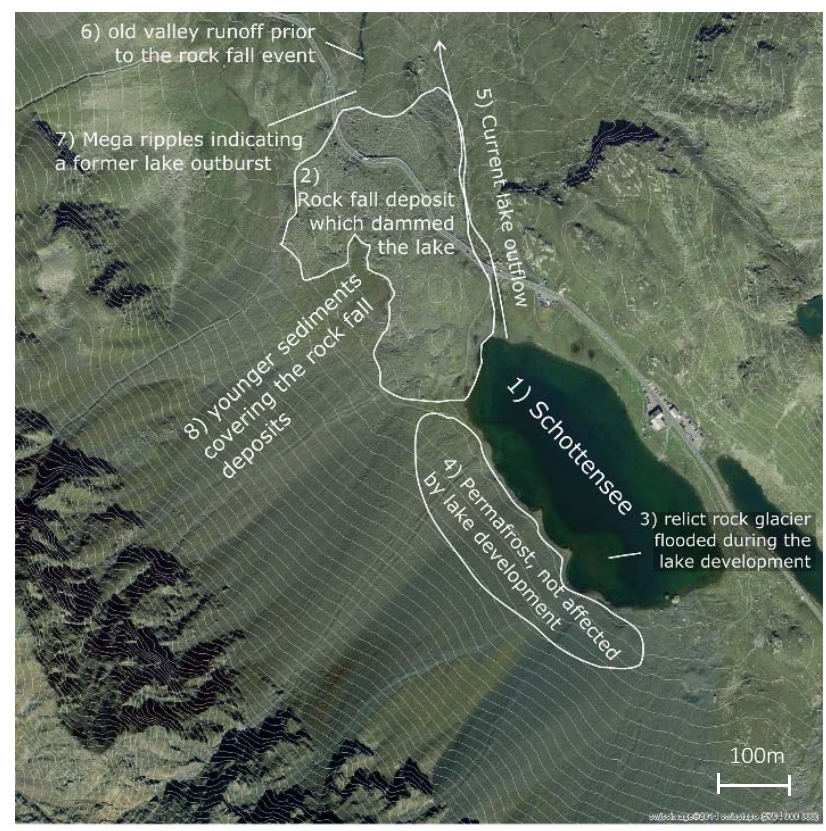

Figure 1: Orthophoto of the Flüelapass. The labelled landforms give in their numbered order a short overview on the geomorphologic history of the site. (orthofoto: swissimage(C2014 swisstopo 5704000 000).

\section{UAV-DATASET}

Aerial images were acquired with a Sony NEX-7 camera (24 Mp, $20 \mathrm{~mm}, \quad \mathrm{~F} / 2.8$ optical lens) mounted on an Ascending Technologies (AscTec) Falcon 8 octocopter (Bühler et al., 2016) in September 2016. About 300 images were taken from a flying height of $100 \mathrm{~m}$ above ground (GSD of $2 \mathrm{~cm}$ ) with an overlap of approximately $75 \%$ along track and $65 \%$ across track. Initial camera positions and orientations were taken from UAV's GNSS- and IMU-system (only heading available in the dataset). Eight ground control points were available, whose coordinates were defined using a Topcon GR5 GNSS receiver in real time kinematic mode.

As Figure 2 and 3 shows, the distribution of control points is far from ideal for a stable geo-referencing of the whole block. However, the region of interest, in that case the slope and the lobe area, is covered sufficiently. Unfortunately, no underwater control points were installed. This limits a stable absolute orientation as well as a rigorous quality control (position and height) of images showing underwater areas mainly.

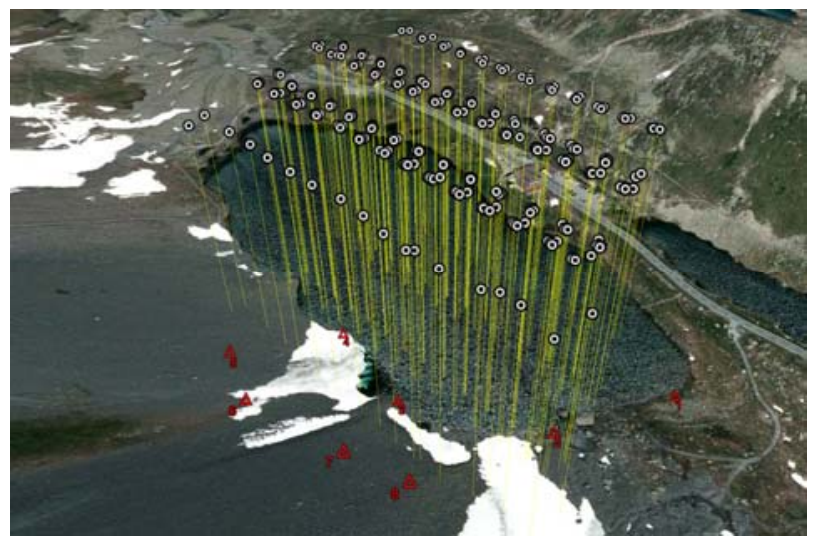

Figure 2. Lake Schottensee with image-positions and control points (CGoogle Earth) 


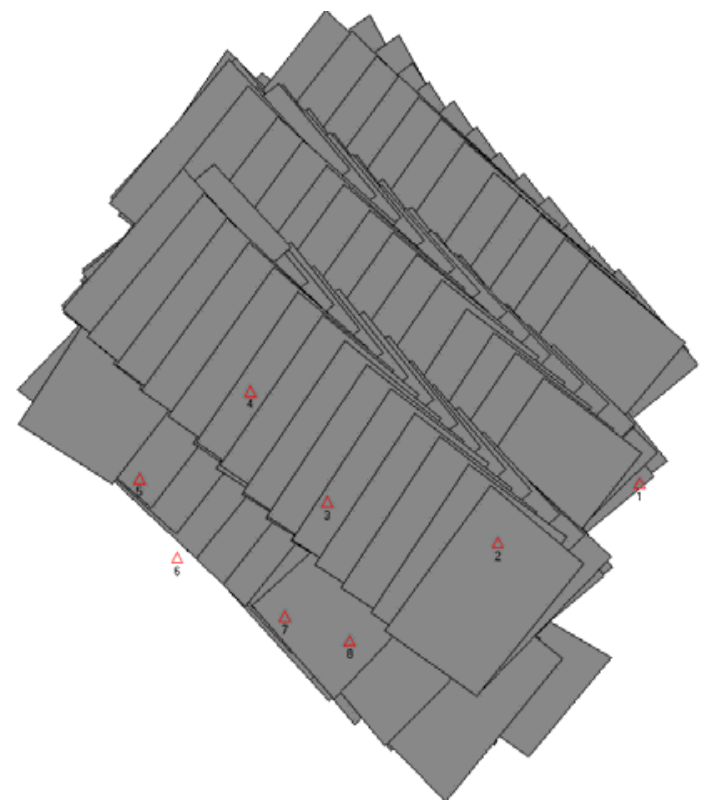

Figure 3. Footprints of images together with control points.

The imaging quality is heterogeneous within the image block. Some images show excessive blurring. Further, the overall brightness varies. This has to be kept in mind when analysing the quality of image matching.

A few months after the UAV-campaign, several underwater check-points were measured via GPS for quality control. A first set was measured through water in November 2016 and a second through ice in December 2016. Figure 4 shows the positions of used checkpoints. Check-points could be measured in situ up to a water depth of $2.8 \mathrm{~m}$.

\section{DATAPROCESSING STRATEGIES}

Finding a suitable strategy for processing the data was a kind of evolutionary process. Starting from the lowest level of complexity, the processing-strategy was refined in order to achieve best results:

- Automated processing in commercial software PhotoScan (4)

- Aerial Triangulation with compensation of refraction (5)

- $\quad$ DEM computation based on triangulation-results and multimedia forward intersection (6)

\section{DATAPROCESSING IN COMMERCIAL SOFTWARE}

At first, the images were oriented in PhotoScan Pro (Agisoft) based on control points and GPS/IMU data. In a next step a georeferenced 3D point cloud of the ground surface as well as a orthophoto-mosaic (Figure 4) was computed in the same software.

The derived DEM is valid for onshore surfaces only, because the software does not take refraction effects into account. As a consequence, the waterbody bottom tends to be lifted up in the DEM. This could be proven by comparing the calculated water depth with the reference measurements acquired in the field (see $6.2)$.

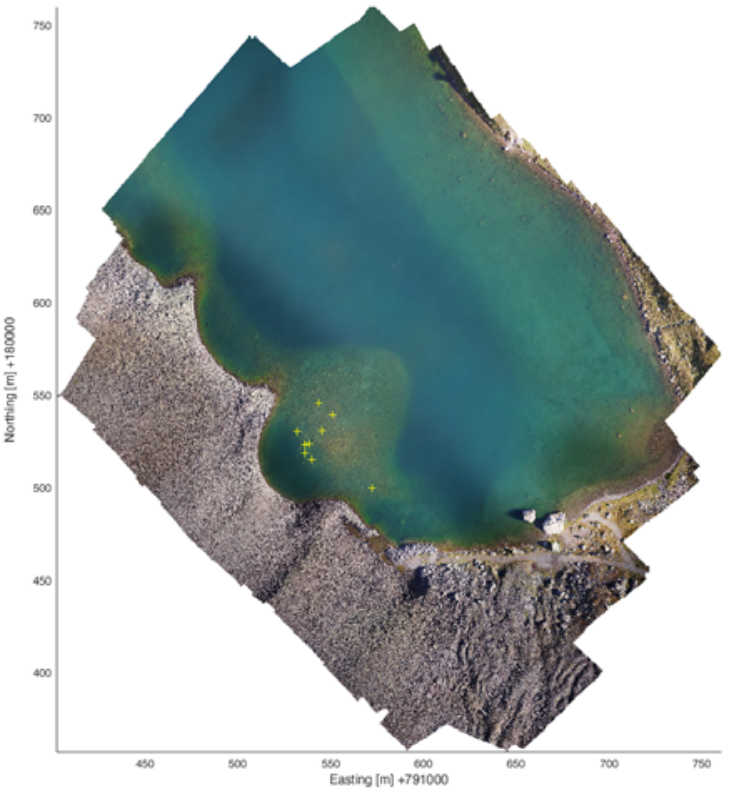

Figure 4: Orthophotomosaic computed in PhotoScan (Agisoft) together with check-points (yellow crosses).

In this specific case, the systematic differences reached values from $\sim 0.3 \mathrm{~m}$ for water depths of about $1.3 \mathrm{~m}$ up to $\sim 0.8 \mathrm{~m}$ for depths of $2.8 \mathrm{~m}$. To avoid this systematic under-estimation of water depth, the refraction has to be considered for underwater points. Unfortunately, all commercial software do not provide this function. Therefore, a dedicated software, which was developed at the Institute for Photogrammetry and Remote Sensing at TU-Dresden (Mulsow, 2010), was used for the processing.

\section{MULTIMEDIA BUNDLE ADJUSTMENT}

As mentioned before, the refraction has to be taken to account when measuring through refracting surfaces. In contrast to the one-media case (usually air), the camera and the object of interest are not in the same optical media. Therefore, the ray between the perspective centre of the camera and an object point is not a straight line. The image ray changes direction while passing the interface between the different medias, following Snell's law. Consequently, the extension of standard photogrammetric imaging models is required.

In aerial photogrammetry, the two-media-problem (air and water) has been discussed since the 1940's. (Rinner, 1948) proposed the stepwise reduction of the problem down to known procedures of standard (one-media) photogrammetry on analogue instruments. First practical aspects of water depths measurements from aerial photographs were highlighted by (Tewinkel, 1963). Several compensation methods for refraction effects were published over the decades, like (Fryer, 1983) or (Butler et.al., 2002). Generally, these methods just add a correction to derived underwater-point coordinates. So, the images had to be orientated separately and the water surface had to be known. First thoughts for an integrated bundle adjustment for multi-media imagery were published by (Kotowski, 1987). (Maas, 2015) presented a multi-media module for planar interfaces which can easily be integrated into photogrammetric standard tools such as spatial resection, spatial intersection or bundle adjustment. 


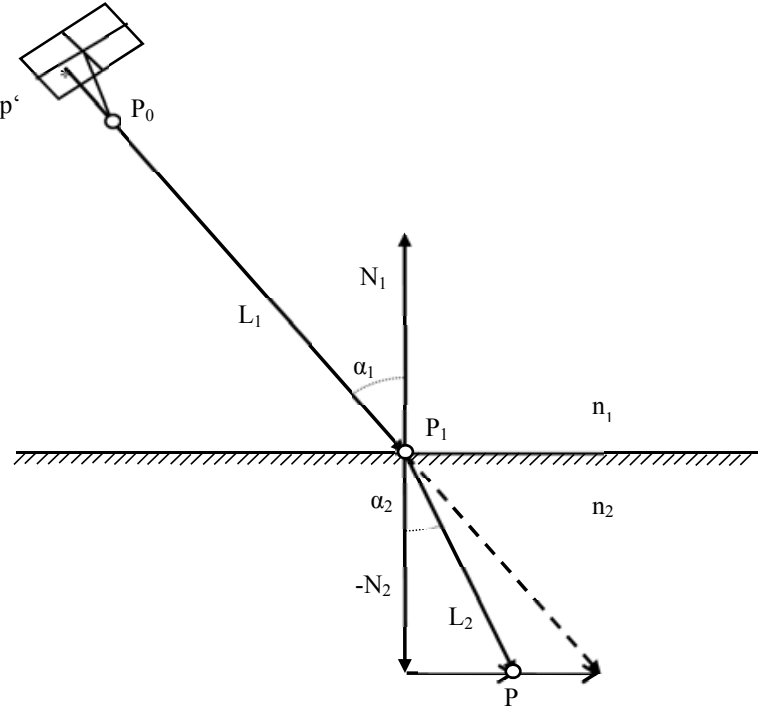

Figure 5: Refraction in two-media case.

An integrated bundle adjustment software was developed by (Mulsow et.al., 2010) based on the work of (Kotowski, 1987). In Kotowski's universal model, the coordinates of the refraction point nearest to the camera ( $\mathrm{P}_{1}$ in Figure 5$)$ defines the image ray together with the projection center $\mathrm{P}_{0}$ and the image point $\mathrm{p}$ ' on the sensor.

The main task in this approach is the complete reconstruction of the image ray path through two or more optical media with different refractive indices. The main advantages of this solution are its universality and flexibility as well as the possibility to implement it into conventional bundle adjustment. The implemented multimedia-bundle was applied to several tasks (Mulsow et.al., 2010; Mulsow et.al.,2014a), where the method could prove its main advantage: as in a conventional bundleadjustment, all parameters (interior and exterior orientation, newpoint coordinates) can be treated as unknowns. Additionally, the surface parameters of interfaces between different media, newpoints in other media as well as refraktive indices can be computed in one integrated adjustment. An in-depth description of the mathematical model can be found in (Mulsow et.al., 2010) and (Mulsow, 2016).

\section{PROCESSING OF UAV-DATA}

First of all, the option of simply using the orientations computed in PhotoScan together with matched image-points will be discussed (6.1). A forward intersection procedure with refraction-compensation sounds feasible. However, it will be shown, that the PhotoScan orientation parameters cannot be used when aiming highest accuracy. Therefore, the images were oriented inside a multimedia bundle adjustment (6.2). The strategy for extraction of a dense DEM will be presented in 6.3 .

\subsection{Forward intersection}

The following approach is applicable if the image orientations are well known in advance. Theoretically, the PhotoScan computation can provide the image-orientations for a straightforward determination of coordinates of underwater points. In that case, the problem can be reduced to a simple forwardintersection task. The correspondent image measurements can be transferred into object space by raytracing. In a first step, the image ray is projected into the object space based on known orientations. In a second step, the image ray has to be intersected with the water surface. Thanks to GPS measurements of absolute water level height and the levelling behaviour of quiet water, the surface parameters are known (plane, surface normal in plumbline direction). The refraction index for water can be extracted from empirical tables.

In the piercing point, the direction-change of the refracted image ray can be computed after the following simple formula (Glassner, 1989) which was derived from Snell's law:

$$
L_{2}=\frac{L_{1}}{\boldsymbol{n}}+\left(\frac{C}{\boldsymbol{n}}-\sqrt{1+\frac{1}{\boldsymbol{n}^{2}}\left(C^{2}-1\right)}\right) N_{1}
$$

where

$$
C=-N_{1} \cdot L_{1} \quad, \quad \boldsymbol{n}=\frac{n_{2}}{n_{1}}
$$

in which: $\mathrm{L}_{1}=$ normalized incoming direction vector

$$
\begin{aligned}
& \mathrm{L}_{2}=\text { refracted direction vector (not normalized) } \\
& \mathrm{N}_{1}=\text { surface normal vector of } \mathrm{T}_{\mathrm{t}} \text { in } \mathrm{P}_{\mathrm{t}} \\
& \mathbf{n}=\text { relative refractive index }
\end{aligned}
$$

So, for each corresponding image measurement an image-ray can be reconstructed inside the water. In a final step, the corresponding image-rays have to be intersected in order to determine the $3 \mathrm{D}$ coordinates of the underwater point. The accuracy can be estimated from the nearest distance of corresponding image-vectors.

In our case however, it turned out that the image orientations calculated in PhotoScan were clearly distorted by refraction effects. Also camera parameters were affected as the radial distortion correlates with refraction if the viewing direction is close to normal to the refractive surface and the distance between the camera positions and the surface has little variation (Freyer et.al., 1986).

Therefore, it was necessary to process the whole data via the multimedia bundle in order to archive best results for onshore and underwater-points.

\subsection{Multimedia bundle adjustment}

In a first step, the number of images was reduced to a feasible level. Blurred images as well as overexposed and underexposed material were sorted out. Further, only images covering the region of interest (slope and lobe) were held in the block. Figure 6 shows the block-layout for further processing.

The whole block was first processed in LPS 9.3 (ERDAS, Hexagon) in order to obtain corresponding points and initial values for image orientations. Image points were measured automatically, which failed for some deeper areas due to low contrast. For this areas, some additional tie points were measured manually first and then refined by least squares matching (LSM). In a second step, the underwater points were labelled manually. Then, the image measurements as well as orientations and camera parameters were transferred from LPS 9.3 to the multimediabundle. A number of 41 images were processed, of which 6 images with water coverage of at least $70 \%$. About 8000 image measurements were handed over for further processing. Images were connected by $\sim 900$ tie points, of which $\sim 150$ were labelled as underwater points. 


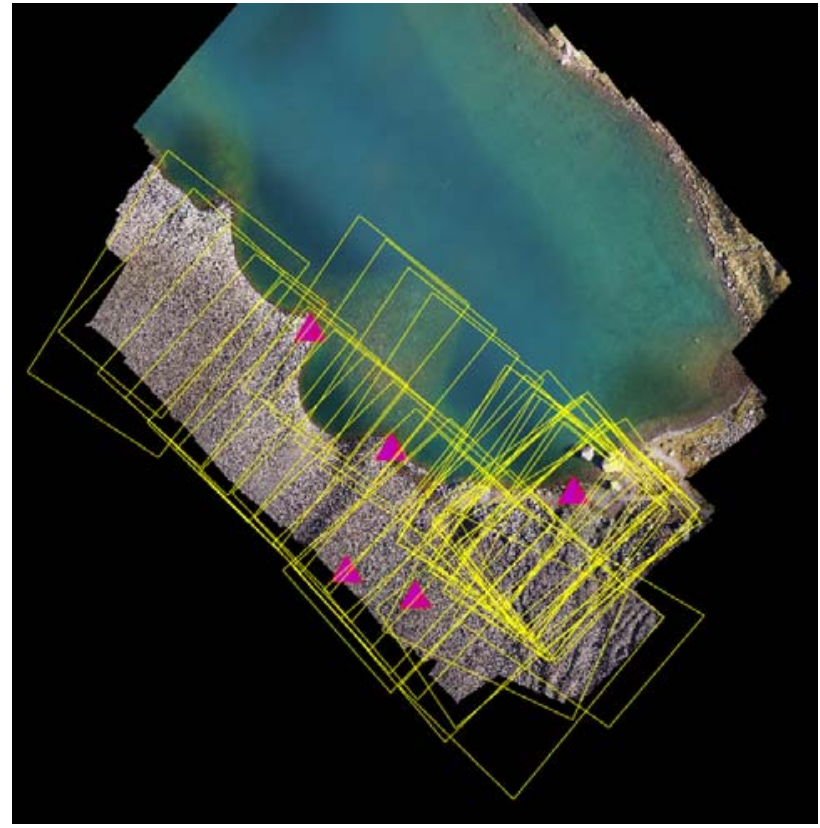

Figure 6: Reduced image block processed in multimediabundle.

Several parameter settings were applied for processing:

I. Adjustment based on all measured image points, all labelled as onshore points

II. Adjustment based onshore points exclusively for camera calibration and image orientation of images with at least $70 \%$ of onshore coverage

III. Adjustment with fixed camera calibration parameters as well as already oriented onshore images (from II) plus remaining un-oriented images ( $>30 \%$ water-coverage), underwater-points together with onshore points

IV. Adjustment with maximum degree of freedom, all image data used, camera parameters as well as orientations were treated as unknowns, image points labelled as onshore points or underwater points.

The simultaneous estimation of water-surface parameters as well as refraction index of water failed due to high correlations between parameters caused by the near-vertical incidence angles of image rays (max angle $\sim 20^{\circ}$ ) and the limited water depth to flight-height ratio.

The compiled results are listed in Table 1. When analysing the quality-parameters of the processing versions, configuration III can be identified as best suited for given data based on the fit of the derived heights with underwater-check points speaks for that parametrization-strategy.

At first glance, the internal height-precision of the underwater points in object space is best for configuration I. Actually, this high accuracy is caused by the refraction effect which is still included in the calculated point-heights here. Not taking the refraction into account, leads to larger intersection angles for image rays of underwater points. When considering refraction, the image ray intersection angle in the denser medium (water) becomes smaller (Maas, 2015), thus degrading the accuracy. As expected, the quality of image-point measurements of underwater points is lower than of onshore points. A degradingfactor of 1.4 can be derived from triangulation-results (parameter setting III).

\begin{tabular}{|c|c|c|c|c|}
\hline \multirow{2}{*}{$\begin{array}{l}\text { Quality } \\
\text { Parameter }\end{array}$} & \multicolumn{4}{|c|}{ Parameter Setting No. } \\
\hline & $\mathbf{I}$ & II & III & IV \\
\hline $\mathbf{s}_{0}[\mathbf{p x}]$ & 0.48 & 0.47 & 0.49 & 0.49 \\
\hline $\begin{array}{l}\text { RMS x' y' } \\
\text { land [px] }\end{array}$ & $0.43 / 0.44$ & $0.42 / 0.43$ & $0.42 / 0.43$ & $0.43 / 0.44$ \\
\hline $\begin{array}{l}\text { RMS x' y' } \\
\text { water[px] }\end{array}$ & $0.44 / 0.43$ & - & $0.51 / 0.51$ & $0.46 / 0.46$ \\
\hline $\mathrm{ck}_{\mathrm{k}}[\mathrm{mm}]$ & 20.443 & 20.467 & 20.467 & 20.441 \\
\hline $\mathbf{x}_{\mathrm{H}}[\mathbf{m m}]$ & -0.0027 & -0.0018 & -0.0018 & -0.0030 \\
\hline $\mathbf{y}_{\mathrm{H}}[\mathbf{m m}]$ & 0.0584 & 0.0552 & 0.0552 & 0.0587 \\
\hline $\begin{array}{l}\text { RMS } \\
\mathrm{X} / \mathrm{Y} / \mathrm{Z} \\
\text { land [cm] }\end{array}$ & $1.7 / 1.4 / 4.4$ & $1.5 / 1.2 / 3.8$ & $1.5 / 1.2 / 3.8$ & $1.7 / 1.4 / 4.4$ \\
\hline \multirow[t]{2}{*}{$\begin{array}{l}\mathrm{RMS} \\
\mathrm{X} / \mathrm{Y} / \mathrm{Z} \\
\text { water }[\mathrm{cm}]\end{array}$} & $1.0 / 1.7 / 4.4$ & - & $0.9 / 1.6 / 5.3$ & $1.0 / 1.8 / 6.0$ \\
\hline & \multicolumn{4}{|c|}{ Underwater check-points } \\
\hline $\begin{array}{l}\text { RMS Z } \\
{[\mathrm{cm}]}\end{array}$ & 2.9 & & 3.5 & 4.0 \\
\hline $\begin{array}{l}\text { RMS [cm] } \\
Z_{\text {target }} Z_{\text {actual }}\end{array}$ & 65.8 & - & 11.8 & 14.5 \\
\hline
\end{tabular}

Table 1. Processing-results for different parameter-settings

\subsection{Underwater DTM determination}

In a first step, image-pairs were defined and transformed into normal images in order to provide some kind of y-parallax-free stereo-images for matching. From theory, the epipolar lines are not straight in multimedia case. However, due to the low water depth the epipolar lines can be seen as straight to a certain degree.

Similar to common matching-procedures, an image-pyramid strategy was implemented. Starting with lowest resolution (reducing-factor 5), points-of-interest were extracted. In order to achieve a good coverage, a raster $(75 \times 50$ cells $)$ was defined for the reference image and for each raster cell the best Harris-point was extracted. These points were searched and measured in the partner-image via LSM (patch size $21 \times 21$, shift in $\mathrm{x}$ direction and one scale parameter only). From matched point pairs, a disparity map was computed. In the next pyramid step, again a raster was defined for the reference image and Harris-points were extracted. Thanks to the disparity map from the previous pyramid step, the search-space can be reduced significantly for matching. The iterative procedure is continued until the finest resolution-level of the image-pyramid is reached. Finally, the matched point-pairs are transferred to the original images and were matched again, but now with a full-parameter set for LSM.

Finally, the image measurements were processed via forward intersection with multimedia compensation as described in 6.1. Image orientation parameters were taken from aerial triangulation configuration III. 


\section{RESULTS}

The main goal of the project was the determination of an underwater DTM of the lobe area. Therefore, the analysis of results focuses on that. In order to quantify the refraction effects on the underwater point height data, two different DTM's were computed - one without and one with refraction compensation. As expected, the water depth was under-estimated when not taking the refraction into account (see Figure 7 and 9). Figure 10 illustrates the differences along a profile. The height offset is between $30-40 \%$. The height accuracy was estimated from check-point data (see Table 1), resulting in a RMS to be $12 \mathrm{~cm}$ (refraction considered). However, for the whole DEM the accuracy is heterogeneous, because of the varying imaging quality which mainly depends on water depth. As Figure 8 shows, structures in shallow water areas were imaged as sharp as onshore structures. However, with increasing water depth, the contrast as well as brightness drops drastically. Points could be successfully measured up to a water depth of ca. $3.5 \mathrm{~m}$ when also accepting some low-quality image points.

For validation purposes, the DTM was intersected with the water level. The derived shore line was projected into the images in order to evaluate its fit. As one can see in Figure 7 and 9, the calculated shore line follows the real line very well

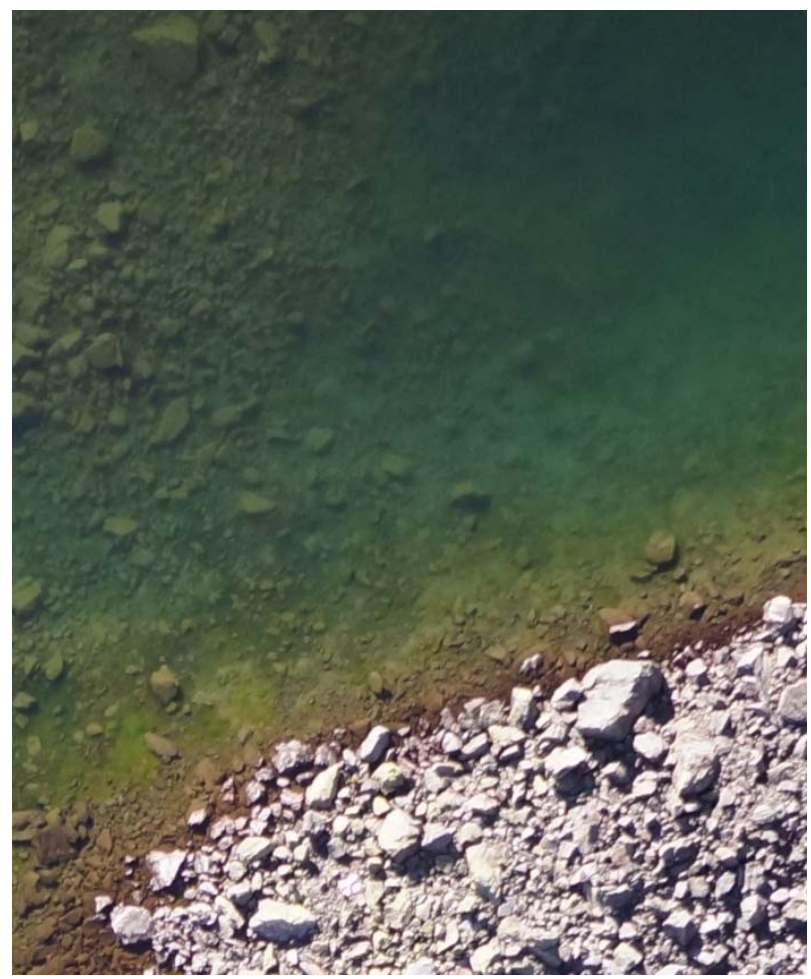

Figure 8: Varying imaging-quality of onshore and underwater areas.

Figure 9: DTM computed via conventional forwardintersection. Note that the depth range was shrinked.

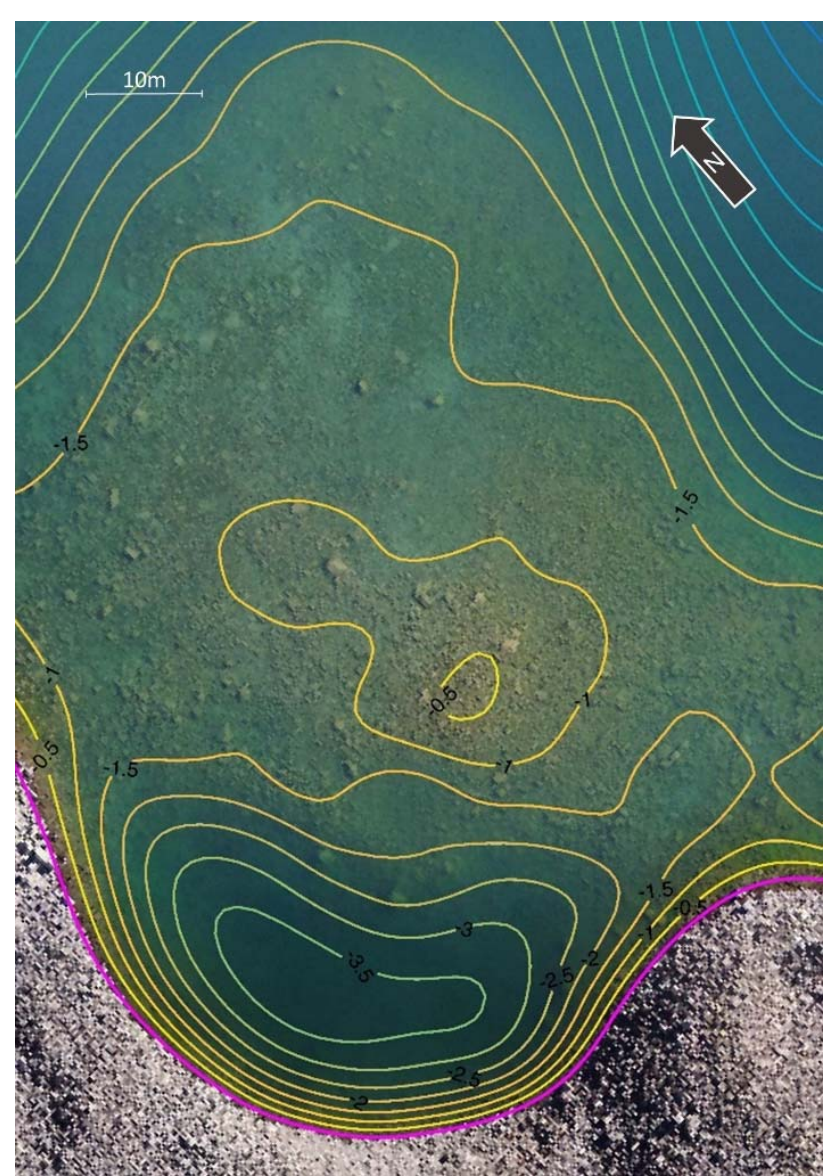

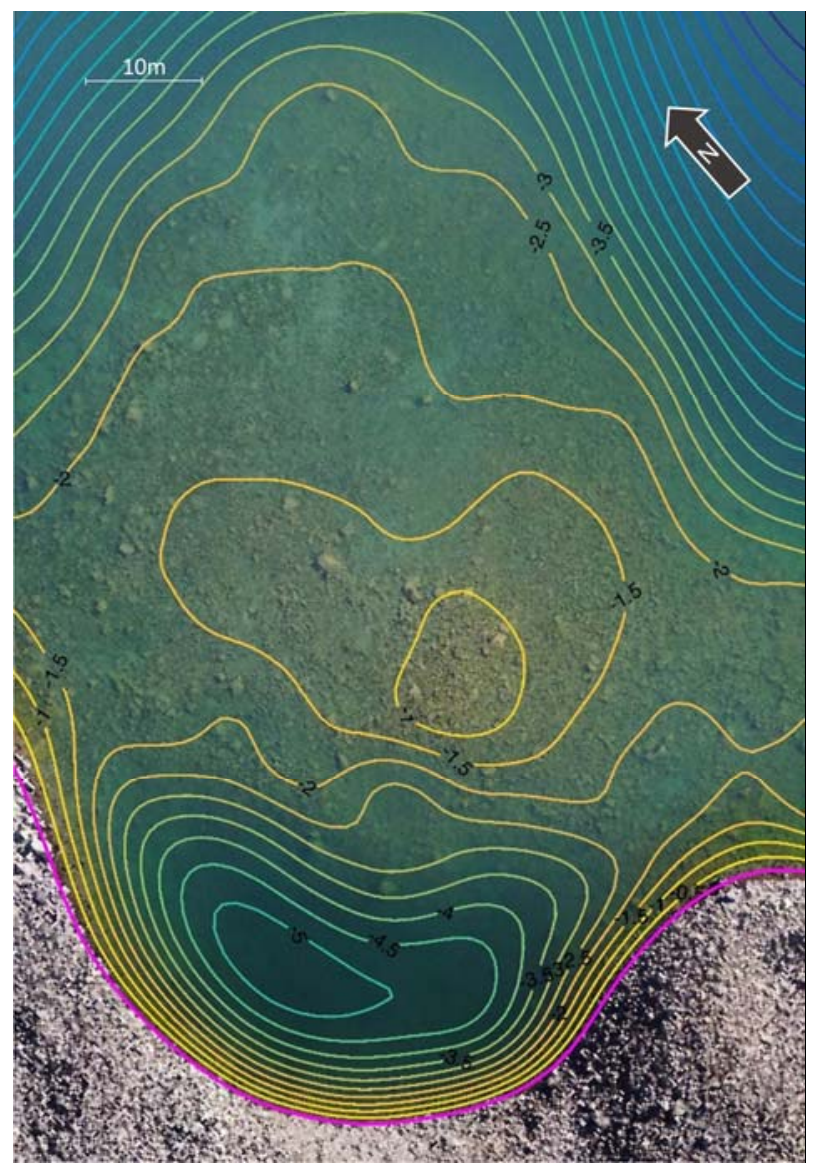

Figure 7: DTM computed via multimedia forward-intersection. The magenta line indicates the shore-line derived from intersection of DEM with water-surface. 


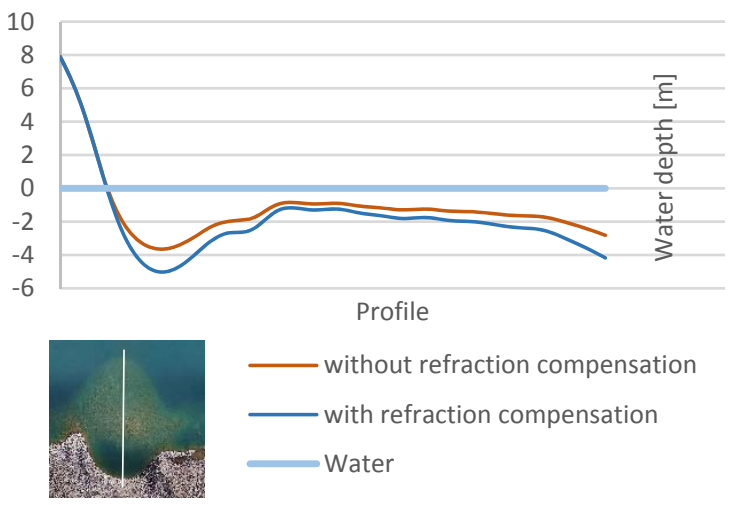

Figure 10: Comparison of DEM heights along a profile.

\section{CONLCUSION AND OUTLOOK}

The paper has shown a photogrammetric workflow for DEM generation from aerial images for regions that contain both land and underwater areas. An appropriate labelling of measured image points and a strict consideration of multimedia geometry in both image orientation and $3 \mathrm{D}$ point coordinate determination turned out to be crucial to achieve good accuracy for underwater points. When neglecting these effects, water depth is significantly underestimated.

A suitable strategy for aerial triangulation has been described. Images showing mainly onshore points should be triangulated first. The derived camera-parameters as well as orientation parameters should be fixed for a second run. In this step, images showing mainly underwater areas should be oriented. The proofof-concept could be provided by processing the data with different parameter-settings. From comparative measurements of check-points, an RMS of $12 \mathrm{~cm}$ for heights of underwater points could be estimated.

During the project, several ideas for improvement arose. First of all, the flight planning should be adjusted for the needs of multimedia photogrammetry. In order to improve the intersectiongeometry, a camera with a larger opening-angle should be applied. Another option could be the use of oblique imagery. However, the camera axis should be tilted only slightly in order to keep the effects of water waves down to a certain level and to prevent total reflection. Furthermore, underwater control points should be installed in order to stabilize the orientation. The shoreline in the images might be extracted automatically by analysing the colour-changes (Kröhnert, et al.,2017), (Mulsow et al., 2014b). To refine the water-land transition of the generated DTM Initial values for the shore line can be derived by intersecting the DTM with the plane water-level, as already shown.

\section{REFERENCES}

Bühler Y, Adams MS, Bösch R, Stoffel A. 2016. Mapping snow depth in alpine terrain with unmanned aerial systems (UASs): potential and limitations. The Cryosphere 10(3): 1075-1088.

Butler, J., Lane, S., Chandler, J. and Porfiri, E., 2002. ThroughWater Close Range Digital Photogrammetry in Flume and Field Environments. The Photogrammetric Record, 17: 419-439. doi:10.1111/0031-868X.00196
Fryer J. G., 1983. Photogrammetry through shallow water. Australian Journal of Geodesy, Photogrammetry and Surveying, 38: $25-38$

Fryer, J.G., Fraser, C.S., 1986. On the calibration of underwater cameras. The Photogrammetric Record, 12 (67), pp. 73-85.

Glassner, A. S., 1989. An Introduction to Ray Tracing. Academic Press, London.

Haeberli W., 1975. Untersuchungen zur Verbreitung von Permafrost zwischen Flüelapass und Piz Grialetsch (Graubünden). Mitteilungen der Versuchsanstalt für Wasserbau, Hydrologie und Glaziologie der ETH Zürich, 17, Zurich, 221 pp

Kenner, R., Phillips, M., Hauck, C., Hilblich, C., Mulsow, C., Buhler, Y., Stoffel, A., Buchroithner, C., 2017. New insights on permafrost genesis and conservation in talus slopes based on observations at Flüelapass, Eastern Switzerland, Geomorphology, 290, 110-113

Kotowski, R., 1987. Zur Berücksichtigung lichtbrechender Flächen im Strahlenbündel. Schriftreihe der DGK, Reihe C, Volume 330.

Kröhnert, M., Meichsner, R., 2017. Segmentation of environmental time lapse image sequences for the determination of shore lines captured by hand-held smartphone cameras. In: ISPRS Annals of the Photogrammetry, Remote Sensing and Spatial Information Sciences IV-2/W4 (2017), S. 1-8

Maas, H.-G., 2015. On the Accuracy Potential in Underwater/Multimedia Photogrammetry. Sensors 2015, 15(8), 18140-18152, doi:10.3390/s150818140, 2015.

Mulsow, C., 2010. A flexible multi-media bundle approach. International Archives of Photogrammetry, Remote Sensing and Spatial Information Sciences, Vol. XXXVIII, Part 5 Commission V Symposium, Newcastle upon Tyne, UK.

Mulsow, C., Maas, H.-G., 2014a. A universal approach for geometric modelling in underwater stereo image processing. Computer Vision for Analysis of Underwater Imagery (CVAUI), ICPR Workshop, Stockholm 2014

Mulsow, C., Koschitzki, R., Maas, H.-G., 2014b. Photogrammetric monitoring of glacier margin lakes. Geomatics, Natural Hazards and Risk.

Mulsow, C., 2016. Ein universeller Ansatz zur MehrmedienBündeltriangulation. In: Photogrammetrie - Laserscanning Optische 3D-Messtechnik, Beiträge der Oldenburger 3D-Tage 2016, Hrsg. Th. Luhmann / Ch. Schumacher, Verlag Herbert Wichmann

Rinner, H., 1948. Problems of two medium photogrammetry. Photogrammetric Engineering, 35(2), pp. 275 - 282.

Tewinkel, G.C., 1963. Water depths from aerial photographs. Photogrammetric Engineering 29 (1963), 1037-1042 